International Journal of Pure and Applied Mathematics

Volume 96 No. 4 2014, 483-505

ISSN: $1311-8080$ (printed version); ISSN: 1314-3395 (on-line version)

url: http://www.ijpam.eu

doi: http://dx.doi.org/10.12732/ijpam.v96i4.5

ijpam.eu

\title{
HIGH ORDER BLOCK IMPLICIT MULTI-STEP (HOBIM) METHODS FOR THE SOLUTION OF STIFF ORDINARY DIFFERENTIAL EQUATIONS
}

\author{
J.P. Chollom ${ }^{1}$, G.M. Kumleng ${ }^{2}$, S. Longwap ${ }^{3}$ \\ $1,2,3$ Department of Mathematics \\ University of Jos \\ Jos, NIGERIA
}

\begin{abstract}
The search for higher order A-stable linear multi-step methods has been the interest of many numerical analyst and has been realized through either higher derivatives of the solution or by inserting additional off step points,supper future points and the likes. These methods are suitable for the solution of stiff differential equations which exhibit characteristics that place severe restriction on the choice of step size. It becomes necessary that only methods with large regions of absolute stability remain suitable for such equations. In this paper, high order block implicit multi-step methods of the hybrid form up to order twelve have been constructed using the multi-step collocation approach by inserting one or more off step points in the multi-step method. The accuracy and stability properties of the new methods are investigated and are shown to yield A- stable methods, a property desirable of methods suitable for the solution of stiff ODEs. The new High Order Block Implicit Multistep methods used as block integrators are tested on stiff differential systems and the results reveal that the new methods are efficient and compete favorably with the state of the art Matlab ode23 code.
\end{abstract}

AMS Subject Classification: 34A45, 65L06

Key Words: block linear integrators, multi step collocation, A-stability, stiff systems, chemical reactions

Received: April 6, 2014

(C) 2014 Academic Publications, Ltd.

$\S$ Correspondence author url: www.acadpubl.eu 


\section{Introduction}

Stiff differential equations have been known to cause singular computational difficulties because of the severe restriction on the step size used. To solve the stiff ODE (1), various authors have made several attempts and came up with various methods of solution.

$$
y^{\prime}=f(x, y), \quad y(0)=0 .
$$

The traditional approach has been the Adams code developed by Shampine [28] where the Adams Moulton formula was used as predictor and Adams Bashfort formula as corrector. Though these yielded a very successful combination but have a draw back because of its requirements for starting values which could lead to growing numerical errors and corrupting further approximations Mayers and Suli[24]. To resolve the issue of starting value, Onimanyi etal[25],[26] proposed the block Linear Multistep methods based on the multi-step collocation approach of Lie and Norset [23] and the self-starting methods of Cash [6]. These methods were developed through the continuous formulation of the linear $\mathrm{k}$-step methods which provided sufficient number of simultaneous discrete methods used as single integrators. However most of these methods were not able to handle stiff ODEs due to stability issues. A popular approach that worked for this class of methods was that by Gear [17],Ascher and Petzold [2] who developed the Backward Differentiation formula of step number $\mathrm{k}$ and order $\mathrm{k}$ with distinguish features where $\mathrm{f}$ is evaluated at the current step $\left[x_{n}, y_{n}\right]$ only. The k-step Adams Moulton methods have failed to perform successfully on stiff equations . Researchers then resorted to implicit Runge-Kutta (IRK) methods, the Backward Differentiation Formulae (BDF) and recently the collocation methods (Hairer and Wanner [19] and Enright [13].These methods have been useful in handling stiff equations due to their better stability properties. To satisfy the A-stability property,the following researchers, Enright [13], Cash [7], [8], [9], Lie and Norsett [23], Chollom [11], Okuonghae and Ikhile [27], Akinfenwa,et al [3] and Ezzeddine and Hojjati [14] developed numerical methods that are A-stable and very suitable for the solution of stiff equations. In this paper, we pursue the hybrid block approach of Chollom and Onumanyi [12] were they constructed block hybrid Adams Moulton methods for $1 \leq K \leq 5$ which produced methods that are shown to possess better stability properties than single integrators and suitable for stiff ODE's. The paper constructed high order block implicit multi-step (HOBIM) methods for $6 \leq K \leq 10$ to advance the integration forward.. The rest of the paper is divided as follows: The derivation of the new methods is done in Section 2, the convergence analysis is in Section 
3 , Numerical experiment to test the efficiency of the new methods is done in Section 4 and the concluding remarks in Section 5

\section{Derivation of the HOBIM Methods}

The high order block implicit multi-step (HOBIM) methods are derived from a class of the multi-step collocation methods with continuous coefficients of the Adams class. These methods in their continuous form are expressed as:

$$
y(x)=\sum_{j=0}^{k} \psi_{j} y_{n+j}-h \sum_{j=0}^{k} \beta_{j} f_{n+j}
$$

for $k$ the step number $k>0, h$ a constant step size given by $h=x_{n+r}-x_{n}, r=$ $1,2, \ldots, k$.The approximation (2) is formulated into the generalization

$$
\bar{y}(x)=\sum_{j=0}^{t-1} \psi_{j} y_{n+j}=h \sum_{j=0}^{s-1} \beta_{j} f(\bar{x}, \bar{y}(\bar{x}))
$$

And is defined over the interval $x \in\left(x_{n+k-1}, x_{n+k}\right)$, where

$$
\Psi_{j}(x)=\sum_{j=0}^{r+m-1} \alpha_{j, j+1} x^{j} h \beta_{j}(x)=h \sum_{j=0}^{r+m-1} \beta_{j, j+1} x^{j}
$$

are the continuous coefficients to be determined satisfying the following interpolation and collocation conditions.

$$
\begin{aligned}
& y\left(x_{n+j}\right)=y_{n+j}, j \in(0,1, \ldots, r-1) \\
& y^{\prime}\left(x_{j}\right)=f_{n+j}, j \in(0,1, \ldots, m-1)
\end{aligned}
$$

where $f_{n+j}=f\left(x_{n+j}, y_{n+j}\right)$. The interpolation and collocation conditions being $r-1$ and $m-1$ respectively .From equations (4) and (5) we have the following imposed conditions:

$$
\begin{aligned}
& \alpha_{j} x_{n+j}=\delta_{i j}, j \in(0,1, \ldots, r-1), i \in(0,1, \ldots, r-1) \\
& h \beta_{j} x_{n+j}=0, j \in(0,1, \ldots, r-1), i \in(0,1, \ldots, r-1) \\
& \alpha_{j}^{\prime} x_{j}=0, j \in(0,1, \ldots, r-1), i \in(0,1, \ldots, r-1) \\
& h \beta_{j}^{\prime} x_{n+j}=\delta_{i j}, j \in(0,1, \ldots, m-1), i \in(0,1, \ldots, r-1)
\end{aligned}
$$

Expressing (6) in matrix form yields the equation

$$
D C=I
$$


I being an identity matrix of order $r \times m$ and the matrix $\mathrm{D}$ given by

$$
D=\left(\begin{array}{ccccccc}
1 & x_{n} & x_{n}^{2} & \ldots & x_{n}^{t} & \ldots & x_{n}^{t+s-1} \\
1 & x_{n+1} & x_{n+1}^{2} & \ldots & x_{n+1}^{t} & \ldots & x_{n+1}^{t+s-1} \\
\vdots & \vdots & \vdots & \vdots & \vdots & \vdots & \vdots \\
1 & x_{n+k-1} & x_{n+k-1}^{2} & \ldots & x_{n+k-1}^{t} & \ldots & x_{n+k-1}^{t+s-1} \\
0 & 1 & 2 x_{n} & \ldots & (t) x_{n} & \ldots & (t+s-1) x_{n} \\
\vdots & \vdots & \vdots & \vdots & \vdots & \vdots & \vdots \\
0 & 1 & 2 x_{s-1} & \ldots & (t) x_{s-1}^{t-1} & \ldots & (t+s-1) x_{s-1}^{(t+s-2)}
\end{array}\right)
$$

is a non-singular matrix of dimension $(\mathrm{s}+\mathrm{t}) \times(\mathrm{s}+\mathrm{t})$. The matrix $\mathrm{C}$ given in $(9)$ and is of

$$
C=\left(\begin{array}{ccccccc}
\alpha_{01} & \alpha_{11} & \ldots & \alpha_{t-1,1} & h \beta_{01} & \ldots & h \beta_{s-1,1} \\
\alpha_{02} & \alpha_{12} & \ldots & \alpha_{t-1,2} & h \beta_{02} & \ldots & h \beta_{s-1,2} \\
\vdots & \vdots & \vdots & \vdots & \vdots & \vdots & \vdots \\
\alpha_{0, t+s} & \alpha_{1, t+s} & \ldots & \alpha_{t-1, t+s} & h \beta_{0, t+s} & \ldots & h \beta_{s-1, t+s}
\end{array}\right)
$$

dimension $(\mathrm{s}+\mathrm{t}) \times(\mathrm{s}+\mathrm{t})$ defined by $C=D^{-1}=C_{i j}, i, j=1, \ldots, s+t-1$ The entries of the matrix $C$ substituted into (4) produces the continuous coefficients of the method referred to as the continuous formulation of the Adams Moulton Class. The continuous interpolant evaluated at both grid and off grid points results in the methods discrete schemes used as block integrators.

\subsection{Derivation of HOBIM $k=6$}

Considering $k=6, x \in\left[x_{n}, x_{n+6}\right]$.The continuous form of the HOBIM method $k=6$ is given by

$$
y(x)=\sum_{j=0}^{k} \psi_{j}(x) y_{n+j}+h \sum_{j=0}^{k} \beta_{j}(x) f_{n+j}+\beta_{j}(\mu), \mu=\frac{11}{2}
$$

Evaluating the matrix (8) with $s=7, t=2$ produces the matrix of the method D in (11)

$$
D=\left(\begin{array}{ccccc}
1 & x_{n+5} & x_{n+5}^{2} & \ldots & x_{n+5}^{8} \\
0 & 1 & 2 x_{n} & \ldots & 8 x_{n}^{7} \\
\vdots & \vdots & \vdots & \vdots & \vdots \\
0 & 1 & 2 x_{n+\mu} & \ldots & 8 x_{n+\mu}^{7} \\
0 & 1 & 2 x_{n+6} & \ldots & 8 x_{n+6}^{7}
\end{array}\right)
$$


Inverting (11) in a Maple environment yields the elements of the matrix $C$ in (9).From the elements of $C$ we obtain the continuous coefficients of the HOBIM method for $k=6$ in (12).

$$
\begin{aligned}
& \alpha \alpha_{5}(x)=1 \\
& h \beta_{0}(x)=\frac{579 \zeta^{2}}{440 h}+\frac{1337 \zeta^{3}}{1485 h^{2}}-\frac{11333 \zeta^{4}}{31680 h^{3}}+\zeta+\frac{679 \zeta^{5}}{7920 h^{4}}-\frac{581 \zeta^{6}}{47520 h^{5}}-\frac{295}{1008 h}-\frac{53 \zeta^{7}}{55440 h^{5}} \\
& -\frac{\zeta^{8}}{31680 h^{7}} \\
& h \beta_{1}(x)=\frac{11 \zeta^{2}}{3 h}-\frac{359 \zeta^{3}}{90 h^{2}}+\frac{2117 \zeta^{4}}{1080 h^{3}}-\frac{191 \zeta^{5}}{360 h^{4}}+\frac{53 \zeta^{6}}{648 h^{5}}-\frac{28025}{18144 h}-\frac{17 \zeta^{7}}{2520 h^{5}}-\frac{\zeta^{8}}{4320 h^{7}} \\
& h \beta_{2}(x)=-\frac{165 \zeta^{2}}{28 h}+\frac{6 \zeta^{3}}{8 h^{2}}-\frac{925 \zeta^{4}}{192 h^{3}}+\frac{347 \zeta^{5}}{24 h^{4}}-\frac{23 \zeta^{6}}{96 h^{5}}-\frac{125}{672 h}-\frac{\zeta^{7}}{48 h^{5}}-\frac{\zeta^{8}}{1344 h^{7}} \\
& h \beta_{3}(x)=\frac{22 \zeta^{2}}{3 h}-\frac{1517 \zeta^{3}}{135 h^{2}}+\frac{1277 \zeta^{4}}{180 h^{3}}-\frac{83 \zeta^{5}}{36 h^{4}}+\frac{11 \zeta^{6}}{27 h^{5}}-\frac{1975}{1008 h}-\frac{47 \zeta^{7}}{1260 h^{5}}-\frac{\zeta^{8}}{720 h^{7}} \\
& h \beta_{4}(x)=\frac{55 \zeta^{2}}{8 h}-\frac{131 \zeta^{3}}{12 h^{2}}-\frac{4169 \zeta^{4}}{576 h^{3}}+\frac{199 \zeta^{5}}{80 h^{4}}-\frac{401 \zeta^{6}}{864 h^{5}}-\frac{125}{756 h}+\frac{5 \zeta^{7}}{112 h^{5}}-\frac{\zeta^{8}}{576 h^{7}} \\
& h \beta_{5}(x)=\frac{33 \zeta^{2}}{5 h}-\frac{107 \zeta^{3}}{10 h^{2}}+\frac{877 \zeta^{4}}{120 h^{3}}-\frac{313 \zeta^{5}}{120 h^{4}}+\frac{61 \zeta^{6}}{120 h^{5}}-\frac{995}{972 h}-\frac{43 \zeta^{7}}{840 h^{5}}+\frac{\zeta^{8}}{489 h^{7}} \\
& h \beta_{\frac{11}{2}}(x)=\frac{55 \zeta^{2}}{8 h}-\frac{131 \zeta^{3}}{12 h^{2}}-\frac{4169 \zeta^{4}}{576 h^{3}}+\frac{199 \zeta^{5}}{80 h^{4}}-\frac{401 \zeta^{6}}{864 h^{5}}-\frac{125}{756 h}+\frac{5 \zeta^{7}}{112 h^{5}}-\frac{\zeta^{8}}{576 h^{7}} \\
& h \beta_{6}(x)=\frac{55 \zeta^{2}}{8 h}-\frac{131 \zeta^{3}}{12 h^{2}}-\frac{4169 \zeta^{4}}{576 h^{3}}+\frac{199 \zeta^{5}}{80 h^{4}}-\frac{401 \zeta^{6}}{864 h^{5}}-\frac{125}{756 h}+\frac{5 \zeta^{7}}{112 h^{5}}-\frac{\zeta^{8}}{576 h^{7}}
\end{aligned}
$$

Substituting (12) into (10) with $\mu=\frac{11}{2}, \zeta=x-x_{n}$ produces the continuous form of the multistep collocation method for $\mathrm{K}=6$ as:

$$
\begin{aligned}
& \bar{y}(x)=y_{n+5}+\left[-\frac{579}{440 h} \zeta^{2}+\frac{1337}{1485 h^{2}} \zeta^{3}-\frac{1133}{31680 h^{3}} \zeta^{4}+\zeta+\frac{679}{7920 h^{4}} \zeta^{5}-\frac{581}{47520 h^{5}} \zeta^{6}\right. \\
& -\frac{295}{1008 h}-\frac{53}{55440 h^{6}} \zeta^{7} \\
& \left.-\frac{1}{31680 h^{7}} \zeta^{8}\right] f_{n}+\left[\frac{11}{3 h} \zeta^{2}-\frac{359}{90 h^{2}} \zeta^{3}+\frac{2117}{1080 h^{3}} \zeta^{4}-\frac{191}{360 h^{4}} \zeta^{5}+\frac{53}{648 h^{5}} \zeta^{6}-\frac{28025}{18144 h}\right. \\
& -\frac{17}{2520 h^{6}} \zeta^{7} \\
& \left.-\frac{1}{4320 h^{7}} \zeta^{8}\right] f_{n}+1+\left[-\frac{165}{28 h} \zeta^{+} \frac{67}{8 h^{2}} \zeta^{3}-\frac{925}{192 h^{3}} \zeta^{4}+\frac{347}{240 h^{4}} \zeta^{\frac{23}{96 h^{5}}} \zeta^{6}-\frac{125}{672 h}+\frac{1}{48 h^{6}} \zeta^{7}\right. \\
& +\left[\frac{22}{3 h} \zeta^{2}-\frac{1517}{135 h^{2}} \zeta^{3}+\frac{1277}{180 h^{3}} \zeta^{4}-\frac{83}{36 h^{4}} \zeta^{5}+\frac{11}{27 h^{5}} \zeta^{6}-\frac{1975}{1008 h}-\frac{47}{1260 h^{6}} \zeta^{7}+\frac{1}{1344 h^{7}} \zeta^{8}\right] f_{n+2} \\
& +\left[-\frac{55}{8 h} \zeta^{2}+\frac{131}{12 h^{2}} \zeta^{3}-\frac{4169}{576 h^{3}} \zeta^{4}+\frac{199}{80 h^{4}} \zeta^{5}-\frac{401}{864 h^{5}} \zeta^{6}-\frac{125}{756 h}+\frac{5}{112 h^{6}} \zeta^{7}\right. \\
& \left.-\frac{107}{10 h^{2}} \zeta^{3}+\frac{877}{120 h^{3}} \zeta^{4}-\frac{313}{120 h^{4}} \zeta^{5}+\frac{61}{120 h^{5}} \zeta^{6}-\frac{995}{972 h}-\frac{43}{840 h^{6}} \zeta^{7}+\frac{1}{576 h^{7}} \zeta^{8}\right] f_{n+4}+\left[\frac{33}{56 h h^{7}} \zeta^{8}\right] f_{n+5} \\
& +\left[\frac{11}{12 h} \zeta^{2}-\frac{1627}{1080 h^{2}} \zeta^{3}\right. \\
& \left.+\frac{3023}{2880 h^{3}} \zeta^{4}-\frac{227}{720 h^{4}} \zeta^{5}+\frac{67}{864 h^{5}} \zeta^{6}-\frac{275}{2016 h}-\frac{41}{5040 h^{6}} \zeta^{7}+\frac{1}{2880 h^{7}} \zeta^{8}\right] f_{n+\frac{11}{2}}+\left[\frac{33}{5 h} \zeta^{2}\right. \\
& \left.+\frac{877}{120 h^{3}} \zeta^{4}-\frac{313}{120 h^{4}} \zeta^{5}+\frac{61}{120 h^{5}} \zeta^{6}-\frac{995}{756 h}-\frac{43}{840 h^{6}} \zeta^{7}+\frac{1}{480 h^{7}} \zeta^{8}\right] f_{n+6} \\
& -\frac{107}{10 h^{2}} \zeta^{3}
\end{aligned}
$$


Table 1: Coefficients of HOBIM $k=6$

\begin{tabular}{cccccccc}
\hline$k$ & 1 & 2 & 3 & 4 & 5 & $\frac{11}{2}$ & 6 \\
\hline$\alpha_{0}$ & 0 & 0 & 0 & 0 & 1 & 0 & 0 \\
$\alpha_{5}$ & 1 & 1 & 1 & 1 & 0 & 1 & 1 \\
$\beta_{0}$ & $\frac{8}{945}$ & $-\frac{78}{36960}$ & $\frac{45}{12474}$ & $-\frac{262}{332640}$ & $\frac{5300}{18144}$ & $-\frac{2335}{15482880}$ & $\frac{18}{90720}$ \\
$\beta_{1}$ & $-\frac{342}{945}$ & $\frac{9789}{36960}$ & $-\frac{550}{12474}$ & $\frac{2475}{332640}$ & $\frac{28025}{18144}$ & $\frac{21150}{15482880}$ & $-\frac{157}{90720}$ \\
$\beta_{2}$ & $-\frac{1224}{945}$ & $-\frac{15939}{36960}$ & $\frac{3861}{12474}$ & $-\frac{11187}{332640}$ & $\frac{3375}{18144}$ & $-\frac{88893}{15482880}$ & $\frac{621}{90720}$ \\
$\beta_{3}$ & $-\frac{6641}{945}$ & $-\frac{41910}{36960}$ & $-\frac{53064}{12474}$ & $\frac{35354}{332640}$ & $\frac{35550}{18144}$ & $\frac{239732}{15482880}$ & $-\frac{1494}{90720}$ \\
$\beta_{4}$ & $-\frac{1224}{945}$ & $-\frac{35904}{36960}$ & $-\frac{148929}{12474}$ & $-\frac{190872}{332640}$ & $\frac{300}{18144}$ & $-\frac{540873}{15482880}$ & $\frac{2496}{90720}$ \\
$\beta_{5}$ & $-\frac{342}{945}$ & $-\frac{2366}{36960}$ & $-\frac{9994}{12474}$ & $-\frac{219285}{332640}$ & $\frac{58735}{18144}$ & $\frac{4566222}{15482880}$ & $\frac{11043}{90720}$ \\
$\beta_{\frac{11}{2}}$ & 0 & $\frac{6656}{36960}$ & $\frac{10240}{12474}$ & $\frac{59904}{332640}$ & $-\frac{12800}{18144}$ & $\frac{3732480}{15482880}$ & $\frac{64000}{90720}$ \\
$\beta_{6}$ & $\frac{8}{945}$ & $-\frac{1023}{36960}$ & $-\frac{1089}{12474}$ & $-\frac{876}{332640}$ & $\frac{2475}{18144}$ & $-\frac{186043}{15482880}$ & $\frac{14103}{90720}$
\end{tabular}

Evaluating (13) at $\zeta=0, h, 2 h, 3 h, 4 h, \frac{11}{2}$, and $6 h$ yields the coefficients of the discrete HOBIM method for $k=6$ used used as block integrator given in Table1.

\subsection{Derivation of HOBIM $k=7$}

Considering $k=7, x \in\left[x_{n}, x_{n+7}\right]$ The continuous form of the method HOBIM $\mathrm{k}=7$ is given by

$$
y(x)=\sum_{j=0}^{k} \psi_{j}(x) y_{n+j}+h \sum_{j=0}^{k} \beta_{j}(x) f_{n+j}+\beta_{j}(\mu), \mu=\frac{13}{2}
$$

Evaluating the matrix (8) with $s=8, t=2$ produces the matrix of the method D in (15)

$$
D=\left(\begin{array}{ccccc}
1 & x_{n+6} & x_{n+6}^{2} & \ldots & x_{n+6}^{9} \\
0 & 1 & 2 x_{n} & \ldots & 9 x_{n}^{8} \\
\vdots & \vdots & \vdots & \vdots & \vdots \\
0 & 1 & 2 x_{n+\mu} & \ldots & 9 x_{n+\mu}^{8} \\
0 & 1 & 2 x_{n+7} & \ldots & 9 x_{n+7}^{8}
\end{array}\right)
$$


Inverting (15) in a Maple environment yields the elements of the matrix $C$ .Substituting the elements of $C$ into (4) produces the continuous coefficients of the method which are substituted into (14) to give the continuous form of the multistep collocation method (16).

$$
\begin{aligned}
& y(x)=y_{n+6}+\left[-\frac{4999}{3640 h} \zeta^{2}+\frac{49213}{49140} h^{2} \zeta^{3}-\frac{5441}{12480 h^{3}} \zeta^{4}+\zeta-\frac{929}{7800 h^{4}} \zeta^{5}-\frac{193}{9360 h^{5}} \zeta^{6}\right. \\
& \left.-\frac{2593}{9100 h}-\frac{1}{455 h^{6}} \zeta^{7}-\frac{23}{17420} h^{7} \zeta^{8}+\frac{1}{294840} h^{8} \zeta^{9}\right] f_{n}+\left[-\frac{91}{22 h} \zeta^{2}-\frac{20}{60} h^{2} \zeta^{3}\right. \\
& +\frac{10301}{3960 h^{3}} \zeta^{4}+\zeta-\frac{31853}{39600} h^{4} \zeta^{5}+\frac{1433}{9504 h^{5}} \zeta^{6}-\frac{3096}{1925 h}-\frac{941}{5440 h^{6}} \zeta^{7} \\
& \left.+\frac{67}{63360 h^{7}} \zeta^{8}-\frac{1}{3540} h^{8} \zeta^{9}\right] f_{n+1}+\left[-\frac{91}{12 h} \zeta^{2}+\frac{1361}{120 h^{2}} \zeta^{3}-\frac{12325}{1728 h^{3}} \zeta^{4}\right. \\
& \left.+\frac{13159}{5400} h^{4} \zeta^{5}-\frac{635}{1296 h^{5}} \zeta^{6}+\frac{33}{70 h}+\frac{439}{7560 h^{6}} \zeta^{7}-\frac{13}{3456} h^{7} \zeta^{8}+\frac{1}{9720} h^{8} \zeta^{9}\right] f_{n+2} \\
& +\left[\frac{65}{6 h} \zeta^{2}-\frac{13177}{756 h^{2}} \zeta^{3}+\frac{143}{12 h^{3}} \zeta^{4}-\frac{1051^{4}}{240 h} \zeta^{5}+\frac{269}{288 h^{5}} \zeta^{6}-\frac{86}{35 h}\right. \\
& \left.-\frac{13}{112 h^{6}} \zeta^{7}+\frac{1}{128 h^{7}} \zeta^{8}-\frac{1}{4536} h^{8} \zeta^{9}\right] f_{n+3}+\left[\frac{91}{8 h} \zeta^{2}+\frac{284}{15 h^{2}} \zeta^{3}-\frac{38989}{2880 h^{3}} \zeta^{4}\right. \\
& \left.+\frac{9409}{1800 h}{ }^{4} \zeta^{5}-\frac{505}{432 h^{5}} \zeta^{6}+\frac{369}{700 h}+\frac{191}{1260 h^{6}} \zeta^{7}-\frac{61}{5760 h^{7}} \zeta^{8}+\frac{1}{3240} h^{8} \zeta^{9}\right] f_{n+4} \\
& +\left[\frac{91}{10 h} \zeta^{2}-\frac{309}{30 h^{2}} \zeta^{3}+\frac{4087}{360 h^{3}} \zeta^{4}-\frac{16313^{4}}{3600 h} \zeta^{5}+\frac{454}{4320 h^{5}} \zeta^{6}-\frac{396}{175 h}\right. \\
& \left.-\frac{713}{5040 h^{6}} \zeta^{7}+\frac{99}{5760 h^{7}} \zeta^{8}-\frac{1}{3240} h^{8} \zeta^{9}\right] f_{n+5}+\left[\frac{91}{12 h} \zeta^{2}+\frac{14087}{1080 h^{2}} \zeta^{3}\right. \\
& -\frac{9371}{960 h^{3}} \zeta^{4}+\frac{2393^{4}}{600 h} \zeta^{5}-\frac{137}{144 h^{5}} \zeta^{6}-\frac{299}{700 h}+\frac{37}{280 h^{6}} \zeta^{7}-\frac{19}{1920 h^{7}} \zeta^{8} \\
& \left.+\frac{1}{3340} h^{8} \zeta^{9}\right] f_{n+6}+\left[\frac{2048}{429 h} \zeta^{2}-\frac{11264}{1365 h^{2}} \zeta^{3}+\frac{120064}{19305 h^{3}} \zeta^{4}-\frac{247552^{4}}{96525 h} \zeta^{5}\right. \\
& \left.+\frac{7168}{11583 h^{5}} \zeta^{6}-\frac{12288}{25025 h}-\frac{11776}{135135 h^{6}} \zeta^{7}+\frac{128}{19305 h^{7}} \zeta^{8}-\frac{256}{1216215} h^{8} \zeta^{9}\right] f_{n+\frac{13}{2}} \\
& +\left[-\frac{13}{14 h} \zeta^{2}+\frac{677}{420 h^{2}} \zeta^{3}-\frac{11}{9 h^{3}} \zeta^{4}+\frac{1829}{3600 h}^{4} \zeta^{5}-\frac{107}{864 h^{5}} \zeta^{6}+\frac{89}{5040 h}\right. \\
& \left.-\frac{11}{8064 h^{6}} \zeta^{7}+\frac{1}{22680 h^{7}} \zeta^{8}-\frac{1}{4536} h^{8} \zeta^{9}\right] f_{n+7} .
\end{aligned}
$$

Evaluating (16) at $\zeta=0, h, 2 h, 3 h, 4 h, 5 h, \frac{13}{2}$ and $7 h$ yields the coefficients of the discrete HOBIM method $\mathrm{k}=7$ in Table2. 
Table 2: Coefficients of HOBIM $k=7$

\begin{tabular}{ccccccccc}
\hline $\mathrm{k}$ & 1 & 2 & 3 & 4 & 5 & 6 & $\frac{13}{2}$ & 7 \\
\hline$\alpha_{0}$ & 0 & 0 & 0 & 0 & 0 & 1 & 0 & 1 \\
$\alpha_{6}$ & 1 & 1 & 1 & 1 & 1 & 0 & 1 & 1 \\
$\beta_{0}$ & $\frac{241725}{31135104}$ & $\frac{528}{467775}$ & $\frac{2167}{3203200}$ & $\frac{1353}{24324300}$ & $\frac{332277}{778377600}$ & $\frac{28523}{100100}$ & $\frac{19090599}{19926466560}$ & $\frac{115467}{778377600}$ \\
$\beta_{1}$ & $-\frac{1111685}{31135104}$ & $-\frac{8328}{467775}$ & $-\frac{24687}{3203200}$ & $-\frac{1996}{24324300}$ & $-\frac{332277}{778377600}$ & $\frac{160992}{100100}$ & $-\frac{187982769}{19926466560}$ & $-\frac{1109667}{778377600}$ \\
$\beta_{2}$ & $-\frac{4075875}{31135104}$ & $\frac{185306}{467775}$ & $\frac{150579}{3203200}$ & $\frac{8148291}{24324300}$ & $\frac{15870569}{778377600}$ & $-\frac{4719}{100100}$ & $\frac{851282003}{19926466560}$ & $\frac{4849559}{778377600}$ \\
$\beta_{3}$ & $-\frac{21503625}{31135104}$ & $\frac{571560}{467775}$ & $-\frac{1522235}{3203200}$ & $-\frac{840840}{24324300}$ & $-\frac{47775585}{778377600}$ & $\frac{245960}{100100}$ & $-\frac{2384941845}{19926466560}$ & $-\frac{12833535}{777877600}$ \\
$\beta_{4}$ & $-\frac{39006825}{31135104}$ & $\frac{376728}{467775}$ & $-\frac{343500}{3203200}$ & $\frac{10470603}{24324300}$ & $\frac{112965567}{778377600}$ & $-\frac{52767}{100100}$ & $\frac{4822595349}{19926466560}$ & $\frac{23326017}{778377600}$ \\
$\beta_{5}$ & $-\frac{27144975}{31135104}$ & $\frac{5578832}{467775}$ & $-\frac{3308877}{3203200}$ & $\frac{28918032}{24324300}$ & $-\frac{476877687}{778377600}$ & $\frac{226512}{100100}$ & $-\frac{8698280019}{19926466560}$ & $-\frac{31923177}{778377600}$ \\
$\beta_{6}$ & $-\frac{22250085}{31135104}$ & $\frac{217338}{467775}$ & $-\frac{1853423}{3203200}$ & $\frac{11821953}{24324300}$ & $-\frac{482889693}{778377600}$ & $-\frac{42757}{100100}$ & $\frac{6050421649}{19926466560}$ & $-\frac{84241443}{778377600}$ \\
$\beta_{\frac{13}{2}}$ & $\frac{6963200}{31135104}$ & $-\frac{32768}{467775}$ & $\frac{442368}{3203200}$ & $-\frac{2080768}{24324300}$ & $\frac{119554048}{778377600}$ & $\frac{49152}{100100}$ & $\frac{46852243456}{19926466560}$ & $-\frac{556285952}{778377600}$ \\
$\beta_{7}$ & $-\frac{1104675}{31135104}$ & $\frac{2904}{467775}$ & $-\frac{60489}{3203200}$ & $\frac{229944}{24324300}$ & $-\frac{16195179}{7778377600}$ & $-\frac{10296}{100100}$ & $-\frac{2146195623}{19926466560}$ & $-\frac{120274869}{778377600}$
\end{tabular}




\subsection{Derivation of HOBIM $k=8$}

Considering $k=8, x \in\left[x_{n}, x_{n+8}\right]$ The continuous form of the method HOBIM $\mathrm{k}=8$ is given by

$$
y(x)=\sum_{j=0}^{k} \psi_{j}(x) y_{n+j}+h \sum_{j=0}^{k} \beta_{j}(x) f_{n+j}+\beta_{j}(\mu), \quad \mu=\frac{15}{2} .
$$

Evaluating the matrix (8) with $s=9, t=2$ produces the matrix of the method $D$ in (18)

$$
D=\left(\begin{array}{ccccc}
1 & x_{n+7} & x_{n+7}^{2} & \ldots & x_{n+7}^{10} \\
0 & 1 & 2 x_{n} & \ldots & 10 x_{n}^{8} \\
\vdots & \vdots & \vdots & \vdots & \vdots \\
0 & 1 & 2 x_{n+\mu} & \ldots & 9 x_{n+\mu}^{8} \\
0 & 1 & 2 x_{n+7} & \ldots & 10 x_{n+7}^{9}
\end{array}\right)
$$

Inverting (18) using the Maple software yields the elements of the matrix C .Substituting the elements of of $\mathrm{C}$ into (17) produces the continuous form of the multistep collocation method for $\mathrm{k}=8$. which are substituted into (14) to give the continuous form of the multistep collocation method. Evaluating the method at $\zeta=0, h, 2 h, 3 h, 4 h, 5 h, 6 h \frac{15}{2}$ and $8 h$ yields the coefficients of the discrete HOBIM method $\mathrm{k}=8$ in Table3.

\subsection{Derivation of HOBIM $k=9$}

Considering $k=9, x \in\left[x_{n}, x_{n+9}\right]$ The continuous form of the method HOBIM $\mathrm{k}=9$ is given by

$$
y(x)=\sum_{j=0}^{k} \psi_{j}(x) y_{n+j}+h \sum_{j=0}^{k} \beta_{j}(x) f_{n+j}+\beta_{j}(\mu), \quad \mu=\frac{17}{2}
$$

Evaluating the matrix (8) with $s=10, t=2$ produces the matrix of the method $D$ in $(20)$

$$
D=\left(\begin{array}{ccccc}
1 & x_{n+8} & x_{n+8}^{2} & \ldots & x_{n+8}^{11} \\
0 & 1 & 2 x_{n} & \ldots & 11 x_{n}^{10} \\
\vdots & \vdots & \vdots & \vdots & \vdots \\
0 & 1 & 2 x_{n+\mu} & \ldots & 11 x_{n+\mu}^{10} \\
0 & 1 & 2 x_{n+9} & \ldots & 11 x_{n+9}^{10}
\end{array}\right)
$$


Table 3: Coefficients of HOBIM $\mathrm{k}=8$

\begin{tabular}{cccccccccc}
\hline $\mathrm{k}$ & 1 & 2 & 3 & 4 & 5 & 6 & 7 & $\frac{15}{2}$ & 8 \\
\hline$\alpha_{0}$ & 0 & 0 & 0 & 0 & 0 & 0 & 1 & 0 & 0 \\
$\alpha_{7}$ & 1 & 1 & 1 & 1 & 1 & 1 & 0 & 1 & 1 \\
$\beta_{0}$ & $\frac{9}{140}$ & $-\frac{12155}{10878368}$ & $\frac{143}{779625}$ & $-\frac{4719}{1601600}$ & $\frac{121}{66237000}$ & $-\frac{65923}{259459200}$ & $\frac{738580}{18528000}$ & $-\frac{6636839}{10218700800}$ & $\frac{148577}{1297296000}$ \\
$\beta_{1}$ & $-\frac{482}{140}$ & $-\frac{192775}{10878368}$ & $-\frac{2200}{779625}$ & $-\frac{54395}{1601600}$ & $-\frac{70}{6237000}$ & $\frac{719279}{259459200}$ & $\frac{44021285}{185328000}$ & $-\frac{71053180}{10218700800}$ & $-\frac{1563925}{1297296000}$ \\
$\beta_{2}$ & $-\frac{1908}{140}$ & $-\frac{4167955}{10878368}$ & $\frac{19340}{779625}$ & $-\frac{303615}{1601600}$ & $-\frac{50}{6237000}$ & $-\frac{3637699}{259459200}$ & $-\frac{708625}{185328000}$ & $\frac{349808000}{10218700800}$ & $\frac{7522385}{1297296000}$ \\
$\beta_{3}$ & $-\frac{774}{140}$ & $-\frac{12451725}{10878368}$ & $-\frac{322190}{779625}$ & $-\frac{1193335}{1601600}$ & $\frac{26730}{6237000}$ & $\frac{11443008}{259459200}$ & $\frac{78863785}{185328000}$ & $-\frac{1055993180}{10218700800}$ & $-\frac{21949785}{1297296000}$ \\
$\beta_{4}$ & $-\frac{2090}{140}$ & $-\frac{8991125}{10878368}$ & $-\frac{931150}{779625}$ & $-\frac{8319025}{1601600}$ & $-\frac{19745}{6237000}$ & $-\frac{25813645}{259459200}$ & $-\frac{34809775}{185328000}$ & $\frac{2218572950}{1021870080}$ & $\frac{43675775}{1297296000}$ \\
$\beta_{5}$ & $-\frac{774}{140}$ & $-\frac{10960235}{10878368}$ & $-\frac{651904}{779625}$ & $-\frac{16382223}{1601600}$ & $\frac{2664442}{6237000}$ & $\frac{48730253}{259459200}$ & $\frac{79718639}{185328000}$ & $-\frac{358811472}{10218700800}$ & $-\frac{63837631}{1297296000}$ \\
$\beta_{6}$ & $-\frac{1908}{140}$ & $-\frac{10750025}{10878368}$ & $-\frac{909700}{779625}$ & $-\frac{17205045}{1601600}$ & $\frac{7431820}{6237000}$ & $-\frac{198188449}{259459200}$ & $-\frac{844343}{185328000}$ & $\frac{5389813880}{10218700800}$ & $\frac{74006075}{1297296000}$ \\
$\beta_{7}$ & $-\frac{482}{140}$ & $-\frac{598095}{10878368}$ & $-\frac{379390}{779625}$ & $-\frac{8700835}{1601600}$ & $\frac{3016750}{6237000}$ & $-\frac{153052471}{259459200}$ & $\frac{41487875}{185328000}$ & $-\frac{31824380500}{10218700800}$ & $\frac{122573165}{1297296000}$ \\
$\beta_{\frac{15}{2}}$ & 0 & $\frac{1392640}{10878368}$ & $\frac{65536}{779625}$ & $\frac{1851392}{1601600}$ & $-\frac{524288}{6237000}$ & $\frac{34816000}{259459200}$ & $-\frac{19152896}{185328000}$ & $-\frac{23519854592}{10218700800}$ & $\frac{988491904}{1297296000}$ \\
$\beta_{8}$ & $-\frac{9}{140}$ & 0 & $-\frac{6985}{779625}$ & $-\frac{231660}{1601600}$ & $\frac{57145}{6237000}$ & $-\frac{4409548}{259459200}$ & $\frac{3343340}{185328000}$ & $\frac{1001060555}{10218700800}$ & $\frac{198229460}{1297296000}$
\end{tabular}


Inverting (20) using the Maple software yields the elements of the matrix C.Substituting the elements of the continuous coefficients into (19) produces the continuous form of the multistep collocation method for $k=9$. Evaluating the continuous form of the linear multi-step method at $\zeta=x-x_{n}, \zeta=$ $0, h, 2 h, 3 h, 4 h, 5 h, 6 h, 7 h, \frac{17}{2}$ and $9 h$ produces the coefficients of the discrete HOBIM method for $k=9$ in Table4 used as block integrators.

\subsection{Derivation of HOBIM $k=10$}

Considering $k=10, x \in\left[x_{n}, x_{n+10}\right]$ The continuous form of the method HOBIM $k=10$ is given by

$$
y(x)=\sum_{j=0}^{k} \psi_{j}(x) y_{n+j}+h \sum_{j=0}^{k} \beta_{j}(x) f_{n+j}+\beta_{j}(\mu), \quad \mu=\frac{19}{2}
$$

Evaluating the matrix (8) with $s=10, t=2$ produces the matrix of the method $D$ in $(22)$

$$
D=\left(\begin{array}{ccccc}
1 & x_{n+9} & x_{n+9}^{2} & \ldots & x_{n+9}^{12} \\
0 & 1 & 2 x_{n} & \ldots & 12 x_{n}^{11} \\
\vdots & \vdots & \vdots & \vdots & \vdots \\
0 & 1 & 2 x_{n+\mu} & \ldots & 12 x_{n+\mu}^{11} \\
0 & 1 & 2 x_{n+9} & \ldots & 12 x_{n+9}^{11}
\end{array}\right)
$$

Inverting [22] using the Maple software yields the elements of the matrix (9).Substituting the elements of the continuous corfficients into(21) produces the continuous form of the multistep collocation method for $k=10$.Evaluating the continuous form of the linear multi-step method at $\zeta=x-x_{n}, \zeta=0, h, 2 h, 3 h, 4 h$, $5 h, 6 h, 7 h, 8 h, \frac{19}{2}$ and $10 h$ produces the coefficients of the discrete HOBIM method for $k=10$ in Table 5 used as block integrators.

\section{Analysis of the New Methods}

In this section, we determine the convergence, construct the regions of absolute stability and obtain the orders of the new HOBIM methods.

\subsection{Convergence Analysis}

Using the approach of Fatunla [15],[16] we determine the convergence of the new HOBIM methods where the block methods are represented as a single block, $r$ 
Table 4: Coefficients of HOBIM $k=9$

\begin{tabular}{ccccccccccc}
\hline $\mathrm{k}$ & 1 & 2 & 3 & 4 & 5 & 6 & 7 & 8 & $\frac{17}{2}$ & 0 \\
\hline$\alpha_{0}$ & 0 & 0 & 0 & 0 & 0 & 0 & 0 & 1 & 0 \\
$\alpha_{8}$ & 1 & 1 & 1 & 1 & 1 & 1 & 0 & 1 & 1 \\
\hline
\end{tabular}

$\beta_{0} \frac{487806319}{83175206400}-\frac{27170}{37437400} \frac{740882}{23289057792}-\frac{6721}{1137161025} \frac{374803}{2395993600}-\frac{322322}{9097288200} \frac{94307785}{582226444800} \frac{310589708}{1137161025} \frac{13779794171}{2980999939737600} \frac{52865527}{582226444800}$ $\beta_{1}-\frac{28178875725}{83175206400} \frac{517808}{37437400}-\frac{102794835}{23289057792} \frac{277134}{1137161025} \frac{4528953}{2395993600} \frac{3464175}{9097288200}-\frac{1109870619}{582226444800} \frac{1958491392}{1137161025}-\frac{159914887275}{2980999939737600} \frac{605909733}{582226444800}$ $\beta_{2}-\frac{11533848400}{83175206400}-\frac{14168242}{37437400} \frac{781136400}{23289057792} \frac{3525324}{1137161025} \frac{25782064}{2395993600}-\frac{16297050}{9097288200} \frac{6053782416}{582226444800}-\frac{616615296}{1137161025} \frac{855986646900}{2980999939737600}-\frac{319063588}{582226444800}$ $\beta_{3}-\frac{41062788312}{8317520400}-\frac{47494668}{37437400} \frac{10314131880}{2328057702} \frac{29081832}{1137161025} \frac{94077048}{2395993600} \frac{41113956}{9097288200}-\frac{20405861736}{58226447273472}-\frac{2809327722708}{1137161025} \frac{810238952984}{588099939737600}$ $\beta_{4}-\frac{131660563034}{83175206400} \frac{27163994}{37437400} \frac{26179560550}{23289057792} \frac{471545932}{1137161025} \frac{267726030}{2395993600}-\frac{37724258}{9097288200} \frac{48123351562}{582226444800}-\frac{3068699920}{1137161025} \frac{6360134317394}{2980999939737600}-\frac{22433058934}{582226444800}$ $\beta_{5}-\frac{39910344474}{83175206400}-\frac{47574670}{37437400} \frac{22441654950}{23289057792} \frac{1356118764}{1137161025}-\frac{1359215858}{2395993600} \frac{189370038}{9097288200}-\frac{86784001590}{582226444800} \frac{5440461312}{1137161025}-\frac{10688638026066}{2980999939737600} \frac{35778539082}{582226444800}$ $\beta_{6}-\frac{111126591576}{83175206400} \frac{28457286}{37437400} \frac{22480234920}{23289057792} \frac{952786692}{1137161025}-\frac{2343736824}{2395993600} \frac{3794299938}{9097288200} \frac{136284991128}{582226444800}-\frac{2232008064}{1137161025} \frac{14402757628116}{2980999939737600}-\frac{43748723304}{582226444800}$ $\beta_{7}-\frac{72986593680}{83175206400}-\frac{45479148}{3743740} \frac{25633436400}{23289057792} \frac{1325517336}{1137161025}-\frac{265033454410905806340}{2395993600} \frac{396653667696}{582226440998016} \frac{18534243061620}{1137161025}-\frac{43998493968}{2980999939737600}$

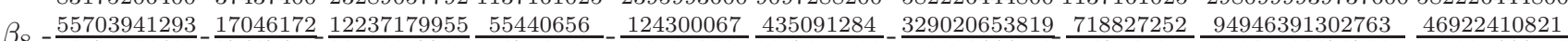
$\beta_{\frac{17}{2}} \frac{154559024896}{83175206400} \frac{2490368}{37437400} \frac{2446458880}{23289057792}-\frac{96206848}{1137161025} \frac{242614272}{2395993600}-\frac{735182848}{9097288200} \frac{69482971136}{582226444800} \frac{620756992}{1137161025} \frac{6734908122764}{2980999939737600} \frac{426040754176}{582226444800}$ $\beta_{9}-\frac{2210729521}{83175206400}-\frac{221221}{37437400} \frac{291294575}{23289057792} \frac{10302578}{1137161025}-\frac{28284685}{2395993600} \frac{77872223}{9097288200}-\frac{8291793367}{582226444800} \frac{1155132160}{1137161025} \frac{2686037350139}{2980999939737600} \frac{88066667689}{58226444800}$ 
Table 5: Coefficients of HOBIM $k=10$

\begin{tabular}{cccccccccccc}
\hline $\mathrm{k}$ & 1 & 2 & 3 & 4 & 5 & 6 & 7 & 8 & 9 & $\frac{19}{2}$ \\
\hline$\alpha_{0}$ & 0 & 0 & 0 & 0 & 0 & 0 & 0 & 0 & 1 & 0 & 0 \\
$\alpha_{9}$ & 1 & 1 & 1 & 1 & 1 & 1 & 1 & 1 & 1 & 1
\end{tabular}

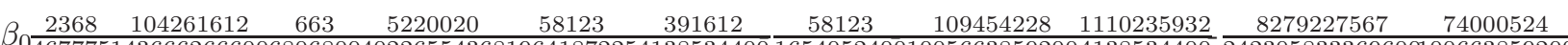

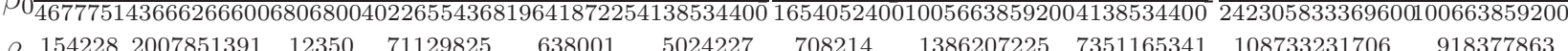
$\beta_{\mathrm{I}} \frac{154228}{4677751436662666006806800402265543681964187225413853440016540524001005663859200} \frac{7351165341}{4138534400} \frac{108733231706}{242305833369600} \frac{918377863}{100663859200}$ $\beta_{2} \frac{6706565461551540}{46777514366626660068068004022655436819641872254138534400} \frac{3942861}{165405240010056633859200} \frac{33375681075}{4138534400} \frac{601900264719}{2423058333696001006638599200}$

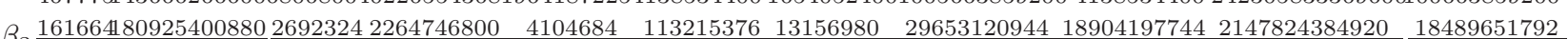
$\beta_{3} \frac{1616775143666266600}{468068004022655436819641872254138534400165405240010056638592004138534400242305833369600} \frac{1840663859200}{100663}$ $\beta_{4} \frac{88819210874516452}{467775143666266600} \frac{839490619708762320}{680680010226554368} \frac{29149458 \quad 30454507}{19641872254138534400} \frac{28558062}{1654052400100566385992004138534400242305833369600100663859200}$ $\beta_{5} \frac{254 \quad 171946522202}{46777514366266600} \frac{52916242442190310 \quad 78356699}{6806800102265543681964187225} \frac{670773454 \quad 37780392 \quad 14135180199429860860978 \quad 9574283911868}{41385348116289654}$ $\beta_{6} \frac{888192128365168386}{467775143666266600} \frac{827203041738269952375517062570955322 \quad 1300806}{680680010226554368196418722541385344001654052400} \frac{2122885490701843694400613407250515126103979593458}{10056638592004138534400242305833369600100663859200}$ $\beta_{7} \frac{16166444402635832}{467775143666266600} \frac{5476380364491675601619218383869697624}{68068004022655436819641872254138534400} \frac{6633686282853124762801927794492015482382099288}{165405240010056638592004138534400242305833369600} \frac{109310215560}{100663859200}$ $\beta_{8} \frac{670656155782312140}{467775143666799814576364130023060949994689459996} \frac{1999438935716323517364}{686753553284} \frac{1742484694411597087548012}{24230583336900100663859200}$ $\beta_{9} \frac{15422876406192317}{36722527420145143399465679732074839273} \frac{780032318 \quad 5475120987317071716457}{7878748729590778 \quad 66987700949}$

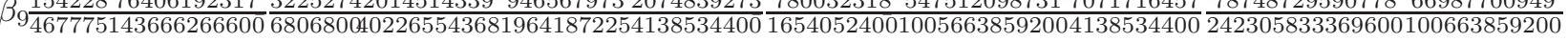
$\beta_{\frac{19}{2}} \quad 0-\frac{15459024896524288}{143666266006806800} \frac{3662807040 \quad 159907840 \quad 376832000127401984 \quad 108213174272}{402265543681964187225413853440016540524001005663859200} \frac{3061186560538550857537536743868334080}{4138534400242305833369600100663859200}$ $\beta_{10} \quad 0 \quad \frac{183756218}{143666266600} \frac{52819 \quad 403964795}{6806800402265543681964187225413853437} \frac{13054249}{165405240010056638592004138534400} \frac{12242558211}{242305833369600100663859200}$ 
point multi-step method of the form

$$
A^{0} y_{m+1}=\sum_{i=1}^{k} A^{i} y_{m+1}+h \sum_{j=0}^{k} B^{i} f_{m-1}
$$

$h$ being a fixed mesh size within a block, $A^{i}, B^{i}, i=0(1) k$ are $r x r$ identity matrix while $Y_{m}, Y_{m-1}$ and $F_{m-1}$ are vectors of numerical estimates.

Definition 1. Zero stability For $n=m r$, for some integer $m \geq 0$, the block method (23) is zero stable if the roots $R_{j}, N=1(1) k$ of the first characteristic polynomial $\rho(R)$ given by

$$
\rho(R)=\operatorname{det}\left[\sum_{i=0}^{k} A^{i} R^{i}\right]=0
$$

satisfies $R_{j} \leq 1$ and for those roots with $R_{j} \leq 1$, the multiplicity nust not exceed two.The block method with coefficients in Table 1 is expressed in the form of (23) gives

$$
\begin{aligned}
& \left(\begin{array}{ccccccc}
1 & 0 & 0 & 0 & -1 & 0 & 0 \\
1 & 0 & 0 & 0 & -1 & 0 & 0 \\
1 & 0 & 0 & 0 & -1 & 0 & 0 \\
1 & 0 & 0 & 0 & -1 & 0 & 0 \\
1 & 0 & 0 & 0 & -1 & 0 & 0 \\
1 & 0 & 0 & 0 & -1 & 0 & 0 \\
1 & 0 & 0 & 0 & -1 & 0 & 0
\end{array}\right)\left(\begin{array}{c}
y_{n+1} \\
y_{n+2} \\
y_{n+3} \\
y_{n+4} \\
y_{n+5} \\
y_{n+6} \\
y_{n+\frac{11}{2}} \\
y_{n+7}
\end{array}\right) \\
& =\left(\begin{array}{lllllll}
0 & 0 & 0 & 0 & 0 & 0 & 0 \\
0 & 0 & 0 & 0 & 0 & 0 & 0 \\
0 & 0 & 0 & 0 & 0 & 0 & 0 \\
0 & 0 & 0 & 0 & 0 & 0 & 0 \\
0 & 0 & 0 & 0 & 0 & 0 & 1 \\
0 & 0 & 0 & 0 & 0 & 0 & 0 \\
0 & 0 & 0 & 0 & 0 & 0 & 0
\end{array}\right)\left(\begin{array}{c}
y_{n-6} \\
y_{n-5} \\
y_{n-4} \\
y_{n-3} \\
y_{n-2} \\
y_{n-1} \\
y_{n}
\end{array}\right) \\
& +h\left(\begin{array}{ccccccc}
-\frac{342}{945} & -\frac{1224}{945} & -\frac{664}{945} & -\frac{1224}{945} & -\frac{342}{945} & 0 & \frac{8}{945} \\
\frac{979}{36960} & -\frac{15030}{36960} & -\frac{41910}{36960} & -\frac{35960}{36960} & -\frac{23661}{36960} & \frac{6656}{36960} & -\frac{1023}{36960} \\
-\frac{550}{124740} & \frac{3861}{124740} & -\frac{53064}{124740} & -\frac{148929}{124740} & -\frac{59994}{124740} & \frac{10240}{124740} & -\frac{1089}{124740} \\
\frac{2475}{332640} & -\frac{11187}{332640} & \frac{35354}{332640} & -\frac{190872}{332640} & -\frac{219285}{332640} & \frac{59904}{332640} & -\frac{8767}{332640} \\
\frac{28025}{18144} & \frac{3375}{18144} & \frac{35550}{18144} & \frac{3000}{18144} & \frac{25785}{18144} & -\frac{12800}{18144} & \frac{2475}{18144} \\
\frac{21150}{15482880} & -\frac{88893}{15482880} & \frac{23972}{15482880} & -\frac{540873}{15482880} & \frac{4566222}{15482880} & \frac{3732450}{15482880} & -\frac{186043}{15482880} \\
-\frac{157}{90720} & \frac{621}{90720} & -\frac{1494}{90720} & \frac{2496}{90720} & \frac{11043}{90720} & \frac{64000}{90720} & \frac{14193}{90720}
\end{array}\right)
\end{aligned}
$$




$$
\times\left(\begin{array}{c}
f_{n+1} \\
f_{n+2} \\
f_{n+3} \\
f_{n+4} \\
f_{n+5} \\
f_{n+\frac{11}{2}} \\
f_{n+6}
\end{array}\right)+h\left(\begin{array}{ccccccc}
0 & 0 & 0 & 0 & 0 & 0 & \frac{8}{945} \\
0 & 0 & 0 & 0 & 0 & 0 & -\frac{78}{36960} \\
0 & 0 & 0 & 0 & 0 & 0 & -\frac{45}{124740} \\
0 & 0 & 0 & 0 & 0 & 0 & -\frac{262}{332640} \\
0 & 0 & 0 & 0 & 0 & 0 & \frac{1062}{18144} \\
0 & 0 & 0 & 0 & 0 & 0 & -\frac{2335}{15482880} \\
0 & 0 & 0 & 0 & 0 & 0 & \frac{18}{90720}
\end{array}\right)\left(\begin{array}{c}
f_{n-6} \\
f_{n-5} \\
f_{n-4} \\
f_{n-3} \\
f_{n-2} \\
f_{n-1} \\
f_{n}
\end{array}\right)
$$

where

$$
\begin{aligned}
A^{0}= & \left(\begin{array}{ccccccc}
1 & 0 & 0 & 0 & -1 & 0 & 0 \\
0 & 1 & 0 & 0 & -1 & 0 & 0 \\
0 & 0 & 1 & 0 & -1 & 0 & 0 \\
0 & 0 & 0 & 1 & -1 & 0 & 0 \\
0 & 0 & 0 & 0 & 1 & 0 & 0 \\
0 & 0 & 0 & 0 & -1 & 1 & 0 \\
0 & 0 & 0 & 0 & -1 & 0 & 1
\end{array}\right), \\
A^{1} & =\left(\begin{array}{lllllll}
1 & 0 & 0 & 0 & 0 & 0 & 0 \\
0 & 1 & 0 & 0 & 0 & 0 & 0 \\
0 & 0 & 1 & 0 & 0 & 0 & 0 \\
0 & 0 & 0 & 1 & 0 & 0 & 0 \\
0 & 0 & 0 & 0 & 0 & 0 & 1 \\
0 & 0 & 0 & 0 & 0 & 0 & 0 \\
0 & 0 & 0 & 0 & 0 & 0 & 0
\end{array}\right)
\end{aligned}
$$

$$
\begin{gathered}
B^{0}= \\
\left(\begin{array}{ccccccc}
-\frac{342}{945} & -\frac{1224}{945} & -\frac{664}{945} & -\frac{1224}{945} & -\frac{342}{945} & 0 & \frac{8}{945} \\
\frac{979}{36960} & -\frac{15030}{36960} & -\frac{41910}{36960} & -\frac{35960}{36960} & -\frac{23661}{36960} & \frac{6656}{36960} & -\frac{1023}{36960} \\
-\frac{550}{124740} & \frac{3861}{124740} & -\frac{53064}{124740} & -\frac{148929}{124740} & -\frac{59994}{124740} & \frac{10240}{124740} & -\frac{1089}{124740} \\
\frac{2475}{332640} & -\frac{11187}{332640} & \frac{35354}{332640} & -\frac{190872}{332640} & -\frac{219285}{332640} & \frac{59904}{332640} & -\frac{8767}{332640} \\
\frac{28025}{18144} & \frac{3375}{18144} & \frac{35550}{18144} & \frac{3000}{18144} & \frac{25785}{18144} & -\frac{12800}{18144} & \frac{2475}{18144} \\
\frac{21150}{15482880} & -\frac{88893}{15482880} & \frac{23972}{15482880} & -\frac{540873}{15482880} & \frac{4566222}{15482800} & \frac{3732450}{15482880} & -\frac{186043}{15482880} \\
-\frac{157}{90720} & \frac{621}{90720} & -\frac{1494}{90720} & \frac{2496}{90720} & \frac{11043}{90720} & \frac{64000}{90720} & \frac{14193}{90720}
\end{array}\right) \\
B^{1}=\left(\begin{array}{cccccccc}
0 & 0 & 0 & 0 & 0 & 0 & \frac{8}{945} & \\
0 & 0 & 0 & 0 & 0 & 0 & -\frac{78}{36960} & \\
0 & 0 & 0 & 0 & 0 & 0 & -\frac{45}{124740} \\
0 & 0 & 0 & 0 & 0 & 0 & -\frac{262}{332640} \\
0 & 0 & 0 & 0 & 0 & 0 & \frac{1062}{18144} \\
0 & 0 & 0 & 0 & 0 & 0 & -\frac{2335}{15482880} \\
0 & 0 & 0 & 0 & 0 & 0 & \frac{18}{90720}
\end{array}\right)
\end{gathered}
$$


Substituting (26) into (24) produces the zero stability polynomial on a parameter $R$ below:

$$
\begin{gathered}
\left.\rho(R)=\operatorname{det}\left[\begin{array}{cccccccc}
1 & 0 & 0 & 0 & -1 & 0 & 0 \\
0 & 1 & 0 & 0 & -1 & 0 & 0 \\
0 & 0 & 1 & 0 & -1 & 0 & 0 \\
0 & 0 & 0 & 1 & -1 & 0 & 0 \\
0 & 0 & 0 & 0 & 1 & 0 & 0 \\
0 & 0 & 0 & 0 & -1 & 1 & 0 \\
0 & 0 & 0 & 0 & -1 & 0 & 1
\end{array}\right)-\left(\begin{array}{ccccccc}
0 & 0 & 0 & 0 & 0 & 0 & 0 \\
0 & 0 & 0 & 0 & 0 & 0 & 0 \\
0 & 0 & 0 & 0 & 0 & 0 & 0 \\
0 & 0 & 0 & 0 & 0 & 0 & 0 \\
0 & 0 & 0 & 0 & 0 & 0 & 1 \\
0 & 0 & 0 & 0 & 0 & 0 & 0 \\
0 & 0 & 0 & 0 & 0 & 0 & 0
\end{array}\right)\right] \\
=\operatorname{det}\left[\begin{array}{ccccccc}
R & 0 & 0 & 0 & -R & 0 & 0 \\
0 & R & 0 & 0 & -R & 0 & 0 \\
0 & 0 & R & 0 & -R & 0 & 0 \\
0 & 0 & 0 & R & -R & 0 & 0 \\
0 & 0 & 0 & 0 & R & 0 & -1 \\
0 & 0 & 0 & 0 & -R & R & 0 \\
0 & 0 & 0 & 0 & -R & 0 & R
\end{array}\right] \\
=R^{6}(R(R-1))=0 \\
\Rightarrow R_{1}=1, R_{2}=R_{3}=R_{4}=R_{5}=R_{6}=0
\end{gathered}
$$

By definition (1), the block method whose coefficients are in Table I is zero stable and of order $p \geq 1$.Therefore, by Henrichi [21],it is convergent. Using a similar approach, the block methods whose coefficients are in Tables $(2,3,4$,and 5) are also convergent.

\subsection{Order of the Methods}

Using the method in Chollom, et al[11] the new HOBIM methods have orders and error constants as shown in Table. 6 below:

\subsection{Regions of Absolute Stability of the HOBIM Methods}

The absolute stability regions of the HOBIM methods are constructed by reformulating the block integrators whose coefficients are in Tables 1-5 as General linear Methods of Butchers [4] using the notations introduced in Burage and Butchers [5].The General linear method (GLM) is represented by a partitioned $(\mathrm{s}+\mathrm{r}) \times(\mathrm{s}+\mathrm{r})$ characterized by the four matrices $A, B, U$ and $V$ expressed in 
Table 6: Order and error constants of the HOBIM methods

\begin{tabular}{|c|c|c|c|c|c|}
\hline method & order & error constant & method & order & error constant \\
\hline HOBIM $\mathrm{k}=6, \mu=\frac{11}{2}$ & $\begin{array}{l}1 \\
1 \\
1 \\
1 \\
1 \\
1\end{array}$ & 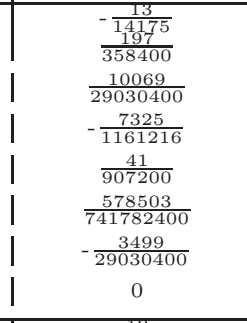 & $\begin{array}{l}\text { новІм } \mathrm{k}=7, \mu=\frac{13}{2} \\
\mid \\
\mid\end{array}$ & $\begin{array}{l}1 \\
1 \\
1 \\
1 \\
1 \\
1\end{array}$ & 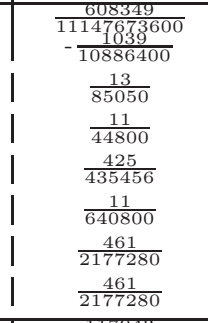 \\
\hline HOBIM $\mathrm{k}=8, \mu=\frac{15}{2}$ & $\begin{array}{ll}1 & \\
1 & \\
1 & 10 \\
1 & \\
1 & \end{array}$ & 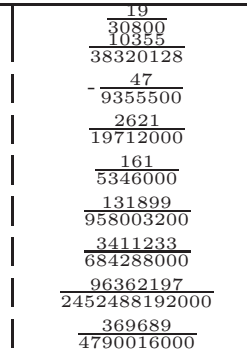 & $\begin{array}{l}\text { HOВIM } \mathrm{k}=9, \mu=\frac{17}{2} \\
\vdots\end{array}$ & $\begin{array}{l}1 \\
1 \\
1 \\
1 \\
1 \\
1 \\
1\end{array}$ & 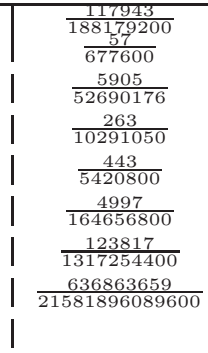 \\
\hline HOBIM $\mathrm{k}=10, \mu=\frac{19}{2}$ & $\begin{array}{ll}1 & \\
1 & \\
1 & 12 \\
1 & \\
1 & \\
1 & \end{array}$ & 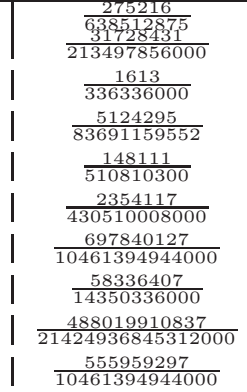 & $\begin{array}{l}1 \\
1 \\
1 \\
1 \\
1 \\
1\end{array}$ & $\begin{array}{l}1 \\
1 \\
1 \\
1 \\
1 \\
1 \\
1 \\
1\end{array}$ & $\begin{array}{l}1 \\
1 \\
1 \\
1 \\
1 \\
1 \\
1 \\
1\end{array}$ \\
\hline
\end{tabular}

the form .

$$
\left[\begin{array}{c}
Y_{[n]}^{1} \\
\vdots \\
Y_{[n]}^{i} \\
\hline Y_{[n]}^{1} \\
\vdots \\
Y_{[n]}^{r}
\end{array}\right]=\left[\begin{array}{l|l}
A & B \\
\hline U & V
\end{array}\right]\left[\begin{array}{c}
h f\left(Y_{[n]}^{1}\right) \\
\vdots \\
h f\left(Y_{[n]}^{i}\right) \\
\left.\hline y_{[n-1]}^{1}\right) \\
\vdots \\
\left.y_{[n-1]}^{r}\right)
\end{array}\right]
$$

The elements of these matrices A,B,U and V are substituted into the recurrence relation

$$
y^{[i-1]}=M(z) y^{[i]}, i=1,2, \ldots, N-1
$$


where

$$
M(z)=U+z B(I-z A)^{-1} V
$$

The stability polynomial

$$
\rho(\eta, z)=\operatorname{det}(\eta I-M(z))
$$

of the method plotted in a matlab environment produces the regions of absolute stability of the HOBIM methods in figures a-e.

\section{Numerical Examples}

To check the behavior of the new HOBIM integrators on Stiff Differential Equations, we solve well known numerical problems using a fix dtep size. The test problems are solved and results obtained are compared with those using ode 23s or exact solutions to illustrate their potential.

Example 4.1 Stiff linear system

$$
\begin{aligned}
y^{\prime}=\left(\begin{array}{cc}
-2 & 1 \\
998 & -999
\end{array}\right) y+\left(\begin{array}{c}
2 \sin x \\
999(\cos x-\sin x)
\end{array}\right), y(0) & =\left(\begin{array}{l}
2 \\
3
\end{array}\right), \\
& 0 \leq x \leq 100, h=0.1
\end{aligned}
$$

Example 4.2 Robertson equation

$$
\begin{aligned}
& y_{1}^{\prime}=-0.04 y_{1}+10000 y_{2} y_{3} \\
& y_{2}^{\prime}=0.04 y_{1}-10000 y_{2} y_{3}-3.0 \times 10^{7} y_{2}^{2}, y(0)=\left(\begin{array}{l}
1 \\
0 \\
y_{3}^{\prime}=3.0 \times 10^{7} y_{2}^{2}
\end{array}\right), 0 \leq x \leq 40, h=0.1
\end{aligned}
$$

Example 4.3 Chemical reaction equation

$$
y^{\prime}=\left(\begin{array}{cc}
-500000.5 & 499999.5 \\
499999.5 & -500000.5
\end{array}\right) y, y(0)=\left(\begin{array}{l}
0 \\
2
\end{array}\right), 0 \leq x \leq 40, h=0.1
$$




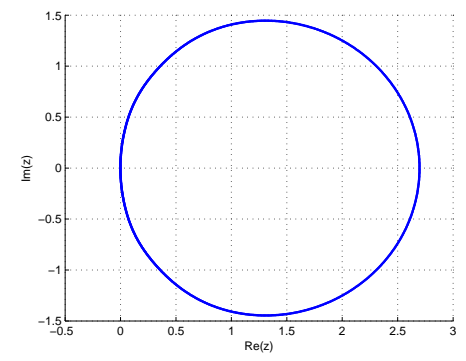

Figure 1: Absolute stability region of HOBIM $\mathrm{k}=6$

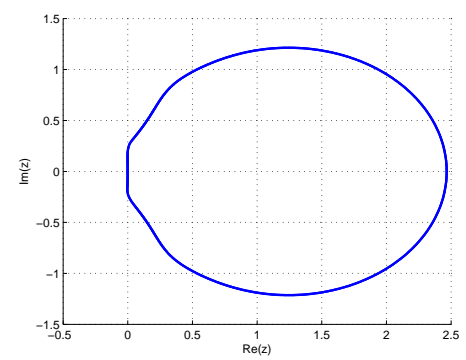

Figure 3: Absolute stability region of HOBIM $\mathrm{k}=8$

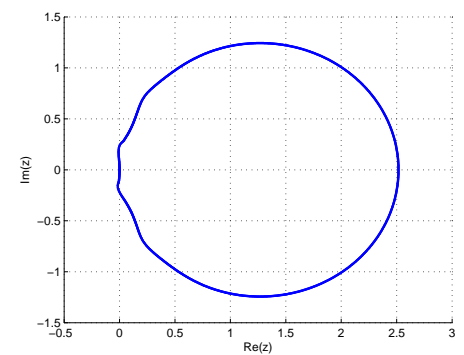

Figure 5: Absolute stability region of HOBIM $\mathrm{k}=10$.

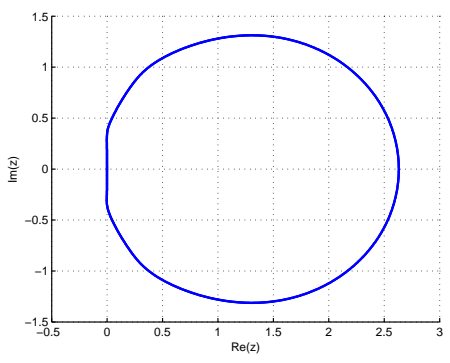

Figure 2: Absolute stability region of HOBIM $\mathrm{k}=7$.

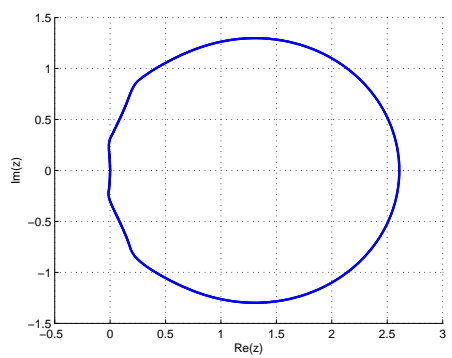

Figure 4: Absolute stability region of HOBIM $\mathrm{k}=9$. 


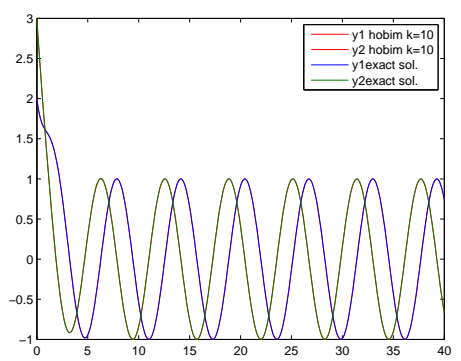

Figure 6: Solution curve of example 4.1

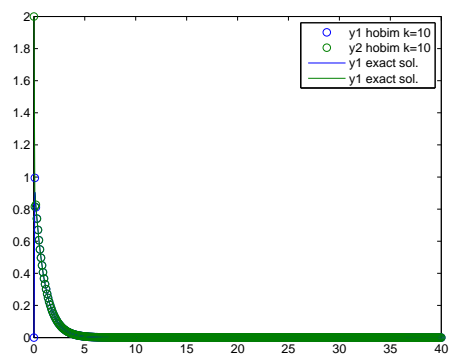

Figure 8: Solution curve of example 4.3

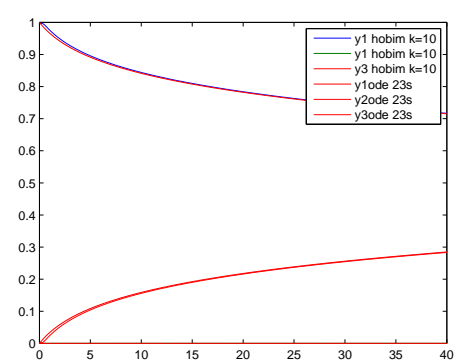

Figure 7: Solution curve of example 4.2

\section{Concluding Remarks}

The search for high order accurate A-stable numerical methods for Stiff Ordinary Differential equations has been pursued since 1952 by various researchers. We have in this paper constructed High Order Block Implicit multi-step (HOBIM) methods for a class of the Adams Moultons family using the multi-step collocation approach. These members are of high order and their regions of absolute stability plotted in figures 1-6 are shown to be A-stable, a property desirable of methods required for the solution of Stiff Ordinary Differential Equations. The new HOBIM member $\mathrm{k}=10$ is tested on the Robertson equation problem 1 used in Aguiar and Ramos[1], a stiff linear equation problem 2 used in Kumleng[22] and a chemical reaction equation problem 3 used in Tahmasbi [29].The result of problem 1 using the HOBIM member $\mathrm{k}=10$ is compared to the results obtained using the inbuilt matlab solver ode23s, the problem 2,stiff linear equation and the chemical reaction equation in problem 3 solved using 
the HOBIM method had their results compared to that of the exact solutions. The solution curves displayed in figures 6,7 and 8 respectively show the efficiency and suitability of the HOBIM methods for the solution of Stiff Ordinary Differential Equations.

\section{References}

[1] J.V.Aguiar and H.Ramos.A family of A-Stable Runge-Kutta collocation methods of higher order for initial-value problems.IMA Journal of Numerical Analysis Advance Access.27(4) 2007

[2] U.Ascher and L. Petzold.Computer method for Ordinary Differential equations and Differential Algebraic equations,SIAM.1998

[3] O.A.Akinfenwa,S.N.Jator and N.M.Yso.A linear Multistep Hybrid Methods with continuousCoefficient for solving Stiff Ordinary Differential Equations.Journal of Modern Mathematics and Statistics,5(2)(2011), 47-53

[4] J.C.Butcher. General Linear Methods for the Parallel Solution of Ordinary Differential Equations in World Scientific Series in Applicable Analysis $.2(1993) .99111$.

[5] K.Burage and J.C.Butcher .Non Linear stability for a general class of differential equations Method,BIT Numerical mathematics, 20(1980),185-203

[6] J.R.Cash,.1980. On the integration of stiff systems of ODE,s using extended BDF.Numer.Maths.34(1980),235-246

[7] J.R.Cash. Second Derivative extended Backward Differentiation Formula for the Numerical Integration of Stiff Systems. SIAM J. Num. Anal.18(1981), 2136.

[8] J.R.Cash. The Integration of Stiff IVPs in ODEs Using Modified Extended BDF 9(5)(1983), 645657.

[9] J.R.Cash.Efficient Time Integrators in the Numerical Method of Lines.Journal of Computation and Applied Mathematics,183(2),295274,2005

[10] J.P.Chollom. The construction of block hybrid Adams Moulton methods with link to two Step Runge-Kutta methods (Ph.D thesis unpublished, University of Jos)(2005). 
[11] J.P.Chollom,J,N.Ndam and G.M.Kumleng.On some properties of the Block linear multi-step Methods,science World Journal,2(3)(2007),11-17

[12] J.P.Chollom and P.Onumanyi.Variable order A-Stable Adams Moulton type block hybrid methods for the solution of stiff first order ODE,s .Journal of the Mathematical Association of Nigeria,Abacus (31)(2004), 2B,77100

[13] W.H.Enright . Second Derivatives Multi-steps Methods for Ordinary Differential Equations. SIAM J. Num. Anal.11(2),(1974), 321331.

[14] A.K.Ezzedine and G.Hojjati.Third Derivative Multistep Methods for Stiff Systems.International Journal of Nonlinear Science,14(4)(2012),443450.

[15] S.O.Fatunla .Block method for second order ODEs. Int.J. of Comput. Maths,England,41(1- 2),(1991),55-63

[16] S.O.Fatunla .A Class of Block methods for second order Initial Value problems.International Journal of Computer Mathematics,England,55(12 ),(1994),119-133

[17] C.W.Gear.Simultaneous Numerical solution of Differential Algebraic Equations:IEEE transaction on Circuit Theory,18(1) (1971), 89-95.

[18] C.W.Gear .Numerical solution of Ordinary Differential Equations:Is there anything left to do? SIAM Review 23(1)(1981),10-24.

[19] E.Hairer.A Runge-Kutta method of order 10,Int.Journal of applied Maths,21(1),(1978),47-59

[20] E.Hairer and G.Wanner . Solving Ordinary Differential 11 Stiff and Differential- Algebraic Problems. 2nd revised edition, Springer series in Computation Mathematics 14, Springer-Verlag, London and New York.(1996).

[21] P.Henrici.Discrete variable methods for odes.John wiley and sons, New York-USA(1962).

[22] G,M,Kumleng.Continuous Generalized Adams Methods in Block form for the solution of Ordinary Differential Equations,P.hD thesis unpublished,Univ. of Jos,Nigeria (2012).

[23] I.Lie and P.Norsett. Super Convergence for the Multi-Step Collocation. Math.Comp. 52(1989). 6579. 
[24] D.F.Mayers and E.Suli.Introduction to Numerical Analysis, Canbridge University Press 2(2003).

[25] P.Onumanyi,D.O.Awoyemi,S,N,Jatau and U.W. Sirisena.New linear Multistep methods with continuous coefficients for the first order Ordinary Initial Value problems,Journal of Nig.Math.Society,13(1994),.37-51.

[26] P.Onumany,S.N.Jator and U.W.Siriena.Continuous finite difference approximation for solving Differential Equations,Inter.I.Comp.Maths $.72(1)(1999), 15-27$

[27] R.I.Okuonghae and M.N.O.Ikhile. -Stable Linear Multistep Methods for Stiff IVPs in ODE. ActaUniv. Palacki. Fac.Rer. Nat. Mathematica. 50 (1) (2011),73-90.

[28] L.F.Shampine and M.W.Reichelt.The Matlab ODE suit SIAM Journal on scientific Computing.18(1)(1977),1-22.

[29] A.Tahmasbi.Numerical Solution for Stiff Ordinary Differential Equation Systems,International Mathematics Forum,3(15),(2008),703-711. 
